\title{
Simultaneous endoscopic ultrasound-guided hepaticogastrostomy and bridging stenting with partial stent-in-stent method
}

Endoscopic ultrasound-guided hepaticogastrostomy (EUS-HGS) is used increasingly worldwide as an alternative to endoscopic retrograde cholangiopancreatography (ERCP) for biliary drainage. The HGS route is used for drainage in cases of hilar malignant biliary obstruction [1 -4]. However, no studies have reported on drainage of all hilar branches via the HGS route in cases that require stenting of the right anterior and right posterior sectional ducts.

We report on a patient with inoperable gallbladder cancer causing hilar obstruction in whom ERCP was not possible. In a single procedure, we performed EUSHGS and hilar stenting of the right anterior and right posterior sectional ducts using the partial stent-in-stent method ( Video 1).

A 66-year-old woman with unresectable gallbladder cancer had duodenal and biliary obstruction. After placing a metal duodenal stent, we performed EUS-HGS. After puncturing the B3 segment using EUS, bile duct enhancement showed hilar obstruction (Bismuth type IIla) ( Fig. 1).
Therefore, we performed simultaneous drainage of all branches through the HGS route. We inserted guidewires (Visiglide2; Olympus, Tokyo, Japan) into B8 and B6 in parallel. We then inserted the first stent $(8 \times 60 \mathrm{~mm}$ uncovered, self-expandable metal stent [SEMS]; Zeo stent V; ZEON Medical Inc., Kawasaki, Japan) from B8 to the left hepatic duct. We then manipulated the guidewire for the first stent through the stent mesh and into B6, running parallel with the existing B6 guidewire. The deployed first stent was dilated using a balloon dilator (REN, 8-mm wide; Kaneka Medix Corporation, Tokyo, Japan), and then the second stent $(8 \times 60 \mathrm{~mm}$ uncovered SEMS; Zeo stent $\mathrm{V}$ ) was deployed from B6 to the left hepatic duct using a partial stent-instent method ( $\triangleright$ Fig.2). Finally, we inserted the third stent $(8 \times 80 \mathrm{~mm}$ fully covered SEMS; X suit NIR; Olympus) from $\mathrm{B} 3$ to the stomach through the HGS route (-Fig.3). No adverse events occurred during the procedure.

Endoscopy_UCTN_Code_TTT_1AS_2AD
Funding

This work was supported in part by The National Cancer Center Research and Development Fund (31-A-13) and by a grant from The Japanese Foundation for Research and Promotion of Endoscopy (JFE).

\section{Competing interests}

The authors declare that they have no conflict of interest.

The authors

Kosuke Maehara', Susumu Hijioka', Yoshikuni Nagashio ${ }^{1}$, Akihiro Ohba ${ }^{1}$, Yuya Kanai ${ }^{2}$, Takuji Okusaka ${ }^{1}$, Yutaka Saito ${ }^{3}$

1 Department of Hepatobiliary and Pancreatic Oncology, National Cancer Center Hospital, Tokyo, Japan

2 Department of Radiological Technology, National Cancer Center Hospital, Tokyo, Japan

3 Endoscopy Division, National Cancer Center Hospital, Tokyo, Japan

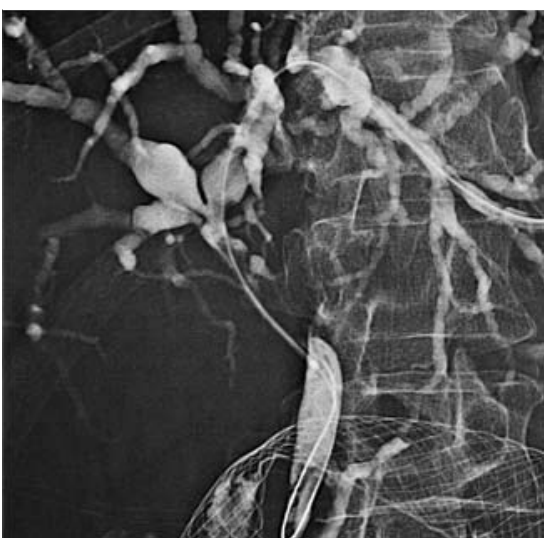

- Fig. 1 Bile duct enhancement via the hepaticogastrostomy route showed hilar malignant biliary obstruction (Bismuth type IIIa). 

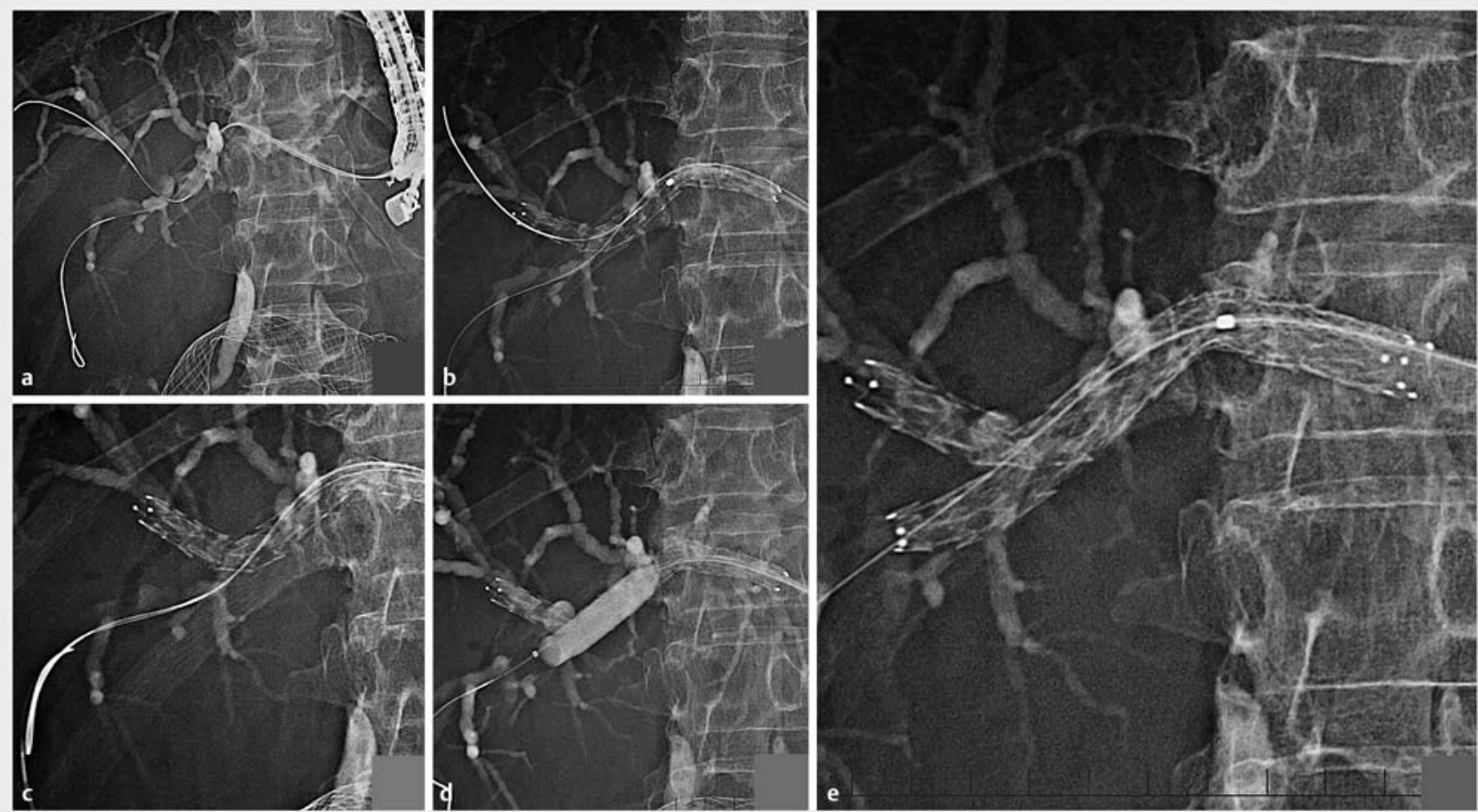

- Fig. 2 Bridging stenting using a partial stent-in-stent method via the hepaticogastrostomy route. a Inserting the guidewires into the B6 and the B8 in parallel. $\mathbf{b}$ Deployment of the first stent from the B8 to the left hepatic duct. c Inserting the guidewire into the B6 from inside the first deployed stent through the stent mesh. $\mathbf{d}$ Balloon dilation of the stricture and stent mesh. e Deployment of the second stent from the B6 to the left hepatic duct using a partial stent-in-stent method.
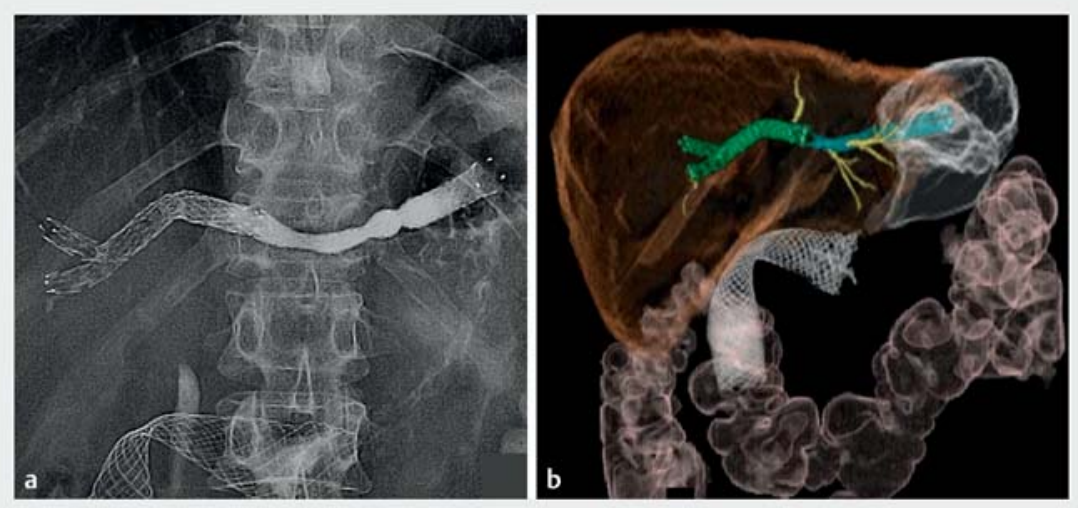

technique in case of proximal biliary obstruction? Therap Adv Gastroenterol 2017; 10: 537-544

[3] Caillol F, Bosshardt C, Reimao S et al. Drainage of the right liver under EUS guidance: a bridge technique allowing drainage of the right liver through the left liver into the stomach or jejunum. Endosc Ultrasound 2019; 8: 199-203

[4] Ogura T, Sano T, Onda S et al. Endoscopic ultrasound-guided biliary drainage for right hepatic bile duct obstruction: novel technical tips. Endoscopy 2015; 47: 72-75

\section{Bibliography}

- Fig. 3 Simultaneous drainage of all branches was accomplished using endoscopic ultrasound-guided hepaticogastrostomy and bridging stenting with a partial stent-in-stent method. a In fluoroscopy. $\mathbf{b}$ In $3 \mathrm{D}$ reconstruction.

Corresponding author

\section{Susumu Hijioka, MD}

Department of Hepatobiliary and Pancreatic Oncology, National Cancer Center Hospital, 5-1-1 Tsukiji, Chuo-ku, Tokyo, Japan Fax: +81-3-35423815

shijioka@ncc.go.jp

\section{References}

[1] Nakai $\mathrm{Y}$, Kogure H, Isayama $\mathrm{H}$ et al. Endoscopic ultrasound-guided biliary drainage for unresectable hilar malignant biliary obstruction. Clin Endosc 2019; 52: 220-225

[2] Moryoussef F, Sportes A, Leblanc S et al. Is EUS-guided drainage a suitable alternative
DOI https://doi.org/10.1055/a-1134-4482

Published online: 15.4.2020

Endoscopy 2020; 52: E381-E382

(c) Georg Thieme Verlag KG

Stuttgart · New York

ISSN 0013-726X 